\title{
Incidental seminal vesicle amyloidosis observed in diagnostic prostate biopsies-are routine investigations for systemic amyloidosis warranted?
}

\author{
Zichu Yang ${ }^{1}$, Alexander Laird ${ }^{2}$, Ashley Monaghan ${ }^{2}$, Morag Seywright ${ }^{3}$, Imran Ahmad $^{2,4}$ and Hing Y Leung, \\ Seminal vesicle (SV) amyloidosis is a well-documented histological entity, but it is observed infrequently. Its incidence is on the rise, \\ which is probably related to the increasing use of prostate biopsies to investigate patients with elevated serum prostate-specific antigen \\ levels. Here, we report seven cases of incidental SV amyloidosis over a 3-year period and consider their relationship to the previously \\ suggested aetiological factors. Based on our series, we conclude that incidental localized SV amyloidosis observed in diagnostic \\ prostate biopsies does not warrant formal investigations for systemic amyloidosis.
}

Asian Journal of Andrology (2013) 15, 149-151; doi:10.1038/aja.2012.125; published online 10 December 2012

Keywords: accessory sex organs; male; prostate biopsies; prostate cancer; prostate diseases; prostatitis; seminal vesicle amyloidosis

\section{INTRODUCTION}

Diagnostic prostate biopsies are commonly performed in men suspected of having prostate cancer and are typically prompted by elevated serum prostate-specific antigen (PSA) levels. The protocol for transrectal ultrasonography (TRUS)-guided prostate biopsies is well developed, and $10-12$ cores are typically obtained. ${ }^{1}$ For patients with initially negative biopsies, the following options are often considered: (i) a period of PSA monitoring; (ii) early repeat prostate biopsies (1012 cores); (iii) saturation prostate biopsies under TRUS guidance; and (iv) transperineal (template) mapping of prostate biopsies. With an increasing trend for PSA testing in the asymptomatic male population over 50 years of age, urologists are performing prostate biopsies on an increasing number of patients, and an increasing number of needle cores are being collected at each setting, including that of the template approach for prostate biopsies. ${ }^{2-4}$

Incidental pathological findings in the prostate and the seminal vesicles (SVs) are well described, including SV amyloidosis, a welldocumented histological entity. ${ }^{5} \mathrm{SV}$ amyloidosis is reported to be associated with haematospermia ${ }^{6}$ and prostatitis, particularly in ageing men. ${ }^{5,7}$ Here, we report on a series of patients with SV amyloidosis, and we evaluated whether the implicated clinical associations applied to our patient cohort. We also considered whether formal investigations for systemic amyloidosis in such patients are warranted.

\section{MATERIALS AND METHODS}

Over a period of 3 years (2008-2010), we identified seven cases of SV amyloidosis in men undergoing either diagnostic prostate biopsies or radical prostatectomy. During this study period, approximately 1500 diagnostic prostate biopsies were performed in our institution. The pathology from these seven patients was formally reviewed, and clinical information was collected, including age, presenting serum PSA level, prostate volume (when available), pathological diagnosis from biopsies and Gleason sum scores for patients with prostate cancer. For patients who underwent diagnostic prostate biopsies, the presence of the SVs was entirely incidental. For patients who received radical prostatectomy for early prostate cancer, the SVs were removed en bloc with the prostate, and the histological examination of the SVs represents a routine part of the assessment.

The prostate tissue was examined for significant inflammatory infiltrate. Immunohistochemistry analyses for Congo (or Sirius) red, amyloid $A$, amyloid $P$, immunoglobulin (Ig) light chains $(\chi / \lambda)$ and $\beta_{2}$ microglobulin were performed using the established clinical protocols within our pathology department. The initial presenting symptoms were also reviewed. The results from investigations of systemic amyloidosis were also included, thus excluding the need for any further evaluation of systemic amyloidosis.

\section{RESULTS}

Within the 3-year study period (2008-2010), we identified seven patients with SV amyloidosis; five patients had undergone TRUSguided prostate biopsies (cases 2-6; mean age 55.6 years; range $47-$ 68 years), and two patients had undergone radical prostatectomy (cases 1 and 7; mean age 55.0 years) (Table 1). Two of the seven patients presented with lower urinary tract symptoms (cases 4 and $6)$, and three patients had haematospermia $(n=1)$, haematuria $(n=1)$ or both $(n=1)$ (Table 1$)$.

The observed amyloid deposits were confined to the SVs with a subepithelial stromal distribution. Staining with Congo red and

${ }^{1}$ Institute of Cardiovascular and Medical Sciences, College of Medical, Veterinary and Life Sciences, University of Glasgow, Glasgow, Scotland G12 8QQ, UK; ${ }^{2}$ Department of Urology, Gartnavel General Hospital, NHS Great Glasgow and Clyde, Glasgow, Scotland G12 OYN, UK; ${ }^{3}$ Pathology NHSGGC, Southern General Hospital, Glasgow, Scotland G51 4TF, UK and ${ }^{4}$ The Beatson Institute for Cancer Research, Glasgow, Scotland G61 1BD, UK Correspondence: Professor HY Leung (h.leung@beatson.gla.ac.uk)

Received: 7 September 2012; Revised: 15 October 2012; Accepted: 16 October 2012; Published online: 10 December 2012 
Table 1 Patient demographics and relevant clinical details

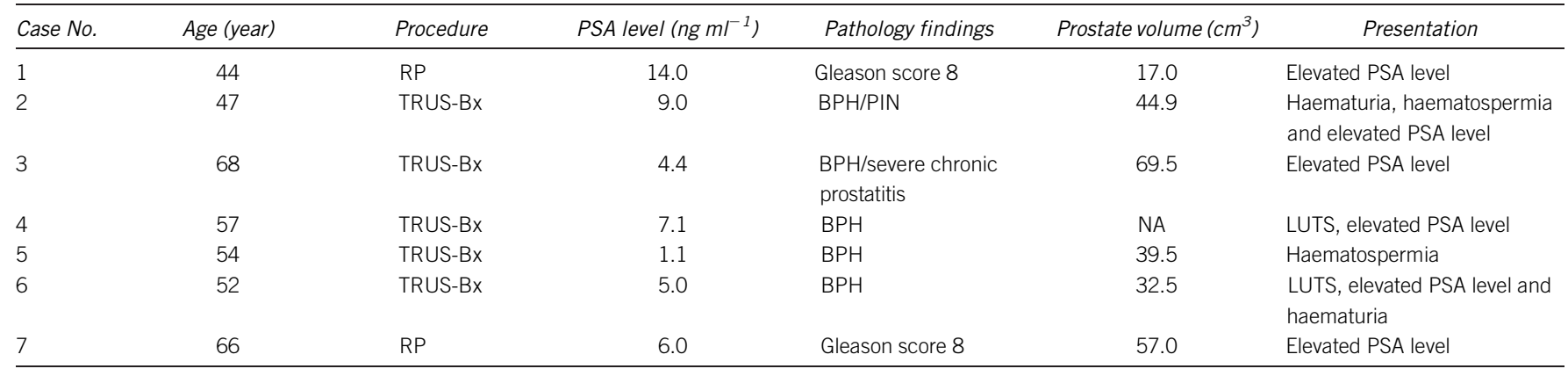

Abbreviations: BPH, benign prostate hyperplasia; LUTS, lower urinary tract symptoms; NA, not available; PIN, prostate intraepithelial neoplasia; PSA, prostate-specific antigen; RP, radical prostatectomy; TRUS-Bx, transrectal ultrasonography-guided prostatic biopsy.

Sirius red (Figure 1) was positive with typical apple green birefringence under polarized light. Immunohistochemical staining for amyloid $\mathrm{P}$ was also performed in three cases. No amyloid deposits were observed around blood vessels or within the prostate tissue. To exclude systemic amyloidosis, additional immunohistochemistry analyses were performed for amyloid protein A and Ig light chains (Table 2). None of the samples studied showed any evidence of significant immunoreactivity for amyloid A or Ig light chains.

\section{DISCUSSION}

Amyloidosis signifies a pathological deposition of extracellular fibrillar proteins in organs and tissues. ${ }^{8}$ The incidence of systemic amyloidosis is difficult to define but is probably underestimated, as amyloidosis can be undiagnosed or misdiagnosed. ${ }^{8}$ The most common types of systemic amyloidosis are amyloid light-chain (AL) amyloidosis, familial transthyretin-associated (ATTR) amyloidosis and amyloid A (AA) amyloidosis, each of which has a different pattern of fibril composition, along with difference in epidemiology, pathogenesis, clinical features, methods of diagnosis and prognosis. ${ }^{8}$ The amyloid A type typically follows an inflammatory stimulus, for

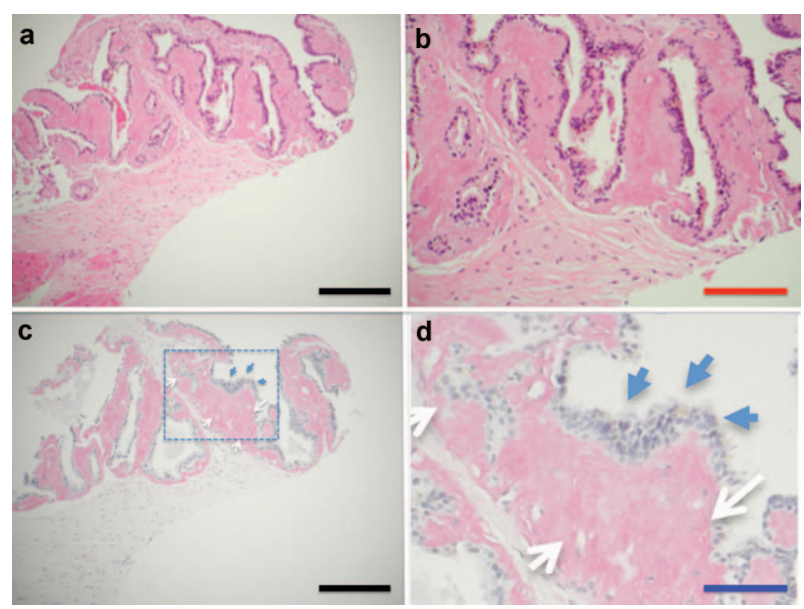

Figure 1 (a-c) Histology from case 5 showing subepithelial deposition of amyloid in the seminal vesicles, Haematoxylin \& Eosin staining (a, b). (c) Strong immunoreactivity for Sirius red confirming the presence of sub-epithelial amyloid deposition (blue arrows signify the epithelium; white arrows highlight the amyloid deposit). (d) Magnified view of the highlighted area in panel (c). Black, red and blue scale bars represent $100 \mu \mathrm{m}, 200 \mu \mathrm{m}$ and $300 \mu \mathrm{m}$ respectively. example, inflammatory diseases such as rheumatoid arthritis or ankylosing spondylitis. The age-adjusted incidence of AL amyloidosis is approximately 5.1 to 12.8 per million person-years. ${ }^{8}$ It can be regarded as myeloma without the bone deposits and is typically associated with abnormal 'free light chains'. The clues to systemic involvement are the involvement of more than one organ (e.g. heart, liver/ spleen and kidney).

Localized amyloidosis of the urogenital tract is an uncommon observation. Within the urinary tract, the bladder is most commonly affected, although the entire urinary tract can be involved..$^{9-12}$ Incidental amyloid deposition within the SVs and ejaculatory system has been reported previously during investigations of diagnostic prostate biopsies. Because the SVs are not routinely included in diagnostic prostate biopsies, we are probably underestimating the incidence of SV amyloidosis. The focus of our report was the clinical significance of this finding, and the optimal protocol for further investigation remains to be objectively defined. ${ }^{5,13}$

Immunohistochemically, localized urogenital amyloidosis is predominantly Ig light chain in origin, with primarily Ig kappa or lambda light chains. ${ }^{13,14}$ While the aetiology of localized urogenital amyloidosis is not fully characterized, chronic inflammation in the affected organ has been suggested as a causative factor. ${ }^{15}$

Here, we reported seven cases of incidental localized amyloidosis in the SVs observed during prostate biopsies or prostatectomy. The symptoms associated with SV amyloidosis are thought to be rare. ${ }^{6,7}$ As expected, six patients in our series presented with elevated PSA levels $\left(>4.0 \mathrm{ng} \mathrm{ml}^{-1}\right)$. Interestingly, among the seven patients in this

\section{Table 2 Summary of the immunohistological analyses}

\begin{tabular}{lllll}
\hline $\begin{array}{l}\text { Patient } \\
\text { No. }\end{array}$ & Congo red & Amyloid A & Amyloid $P$ & $\begin{array}{l}\text { Immunoglobulin light } \\
\text { chains }\end{array}$ \\
\hline 1 & & - & + & $\begin{array}{l}\text { Equivocal } \\
\chi / \lambda \text { light chains weak }+ \\
\beta_{2} \text { microglobulin weak }+\end{array}$ \\
2 & + & - & + & NA \\
3 & + & NA & NA & + \\
4 & + & - & + & Equivocal \\
5 & + & - & NA & Equivocal \\
6 & + & - & NA & - \\
7 & + & - & NA & \\
\hline
\end{tabular}

Abbreviation: NA, data not available (not performed).

+ : positive immunoreactivity detected.

- : negative for staining. 
series, significant inflammatory infiltrate was observed in the biopsies from only one patient (case 3), which does not support the proposed relationship between SV amyloidosis and prostatitis. Overall, the subepithelial stromal distribution within the SVs and the absence of amyloid within the prostate tissue strongly suggest a localized nature of amyloidosis in our cases.

In our case series, the mean age of men with SV amyloidosis was 55.4 years, which argues against the concept of senile SV amyloidosis. Symptoms of either haematuria or haematospermia were documented in only three of the seven patients $(\sim 42.8 \%)$.

The importance of SV amyloidosis as an underlying pathology for haematospermia has recently been reviewed. ${ }^{16}$ It is interesting to note that SV amyloidosis was considered inevitably related to systemic amyloidosis even in that review. Such a perceived risk of systemic involvement in the presence of SV amyloidosis may be overstated. Given the small number of cases reported in this series, no firm conclusions can be drawn. However, our report opens the debate on whether it is appropriate to automatically initiate routine, extensive investigations, including immunohistochemical analyses and patient referral, in patients found to have incidental SV amyloidosis with only one organ affected.

\section{AUTHOR CONTRIBUTIONS}

AL, AM, HYL, MS identified patient cohort; AL, AM, HYL retrieved clinical data; MS carried out pathology review and obtained histology images; IA contributed to the preparation of figure; ZY, HYL wrote the paper.

\section{COMPETING FINANCIAL INTERESTS}

The authors declare no competing financial interests.

\section{ACKNOWLEDGMENTS}

The authors thank Dr John Hunter, Consultant Rheumatologist, for helpful discussions.

1 Heidenreich A, Aus G, Bolla M, Joniau S, Matveev VB et al. EAU guidelines on prostate cancer. Eur Urol 2008; 53: 68-80.

2 Lawrentschuk N, Pan D, Stillwell R, Bolton DM. Implications of amyloidosis on prostatic biopsy. Int J Urol 2004; 11: 925-7.

3 Merrick GS, Taubenslag W, Andreini H, Brammer S, Butler WM et al. The morbidity of transperineal template-guided prostate mapping biopsy. BJU Int 2008; 101: 1524-9.

4 Taira AV, Merrick GS, Galbreath RW, Andreini H, Taubenslag W et al. Performance of transperineal template-guided mapping biopsy in detecting prostate cancer in the initial and repeat biopsy setting. Prostate Cancer Prostatic Dis 2010; 13: 71-7.

5 Pitkanen P, Westermark P, Cornwell GG, 3rd, Murdoch W. Amyloid of the seminal vesicles. A distinctive and common localized form of senile amyloidosis. Am J Pathol 1983; 110: 64-9.

6 Furuya S, Masumori N, Furuya R, Tsukamoto $\mathrm{T}$, Isomura $\mathrm{H}$ et al Characterization of localized seminal vesicle amyloidosis causing hemospermia: an analysis using immunohistochemistry and magnetic resonance imaging. J Urol 2005; 173: 1273-7.

7 Coyne JD, Kealy WF. Seminal vesicle amyloidosis: morphological, histochemical and immunohistochemical observations. Histopathology 1993; 22: 173-6.

8 Falk RH, Comenzo RL, Skinner M. The systemic amyloidoses. N Engl J Med 1997; 337: 898-909.

9 Merrimen JL, Alkhudair WK, Gupta R. Localized amyloidosis of the urinary tract: case series of nine patients. Urology 2006; 67: 904-9.

10 Stillwell TJ, Segura JW, Farrow GM. Amyloidosis of the urethra. J Urol 1989; 141: 52-3.

11 Esslimani M, Serre I, Granier M, Robert M, Baldet P et al. Urogenital amyloidosis: clinico-pathological study of 8 cases. Ann Pathol 1999; 19: 487-91.

12 Tirzaman O, Wahner-Roedler DL, Malek RS, Sebo TJ, Li CY et al. Primary localized amyloidosis of the urinary bladder: a case series of 31 patients. Mayo Clin Proc 2000; 75: 1264-8.

13 Kee KH, Lee MJ, Shen SS, Suh JH, Lee OJ et al. Amyloidosis of seminal vesicles and ejaculatory ducts: a histologic analysis of 21 cases among 447 prostatectomy specimens. Ann Diagn Pathol 2008; 12: 235-8.

14 Fujihara S, Glenner GG. Primary localized amyloidosis of the genitourinary tract: immunohistochemical study on eleven cases. Lab Invest 1981; 44: 55-60.

15 Gertz MA, Rajkumar SV. Localized amyloidosis. In: Amyloidosis: Diagnosis and Treatment. New York: Springer; 2010. p99.

16 Ahmad I, Krishna NS. Hemospermia. J Urol 2007; 177: 1613-8. 